\title{
Heroin-Induced Spongiform Leukoencephalopathy: Value of Diffusion MR Imaging
}

\author{
Cheng-Yu Chen, Kwo-Wei Lee, Chueng-Chen Lee, Shy-Chi Chin, Hsiao-Wen Chung, and \\ Robert A. Zimmerman
}

\begin{abstract}
The diffusion-weighted (DW) magnetic resonance (MR) imaging findings of a patient with subacute stage of heroin-induced vacuolating myelinopathy are reported. The diffuse decrease of apparent diffusion coefficient (ADC) of the white matter on DW imaging is attributed to restricted water diffusion, which is known to be caused by fluid entrapment within the myelin lamellae without demyelination. Index Terms: Heroin intoxication-Myelinopathy-Diffusion-weighted MRI.
\end{abstract}

\section{INTRODUCTION}

Encephalopathy induced by heroin intoxication is characterized by spongiform myelinopathy as a result of fluid accumulation within the myelin sheaths (1). Computed tomography has shown decreased attenuation of cerebral white matter in patients with heroin intoxication (1). Magnetic resonance (MR) imaging typically shows diffuse T2 elongation of white matter due to the underlying pathologic feature of spongiform leukoencephalopathy (2). These imaging findings are, however, not specific for heroin-induced encephalopathy and may resemble other leukoencephalopathies of different etiologies $(3,4)$. The recent use of diffusion-weighted MR (DW) technique has improved the understanding of the pathophysiology of white matter myelination, dysmyelination, and demyelination by showing the anisotropically restricted diffusion of water inherent in the axons $(5,6,7)$.

We report a case of heroin intoxication in which DW MR images showed diffuse isotropic restriction of water diffusion in white matter at the intermediate stage of disease. The spongiform myelinopathy revealed by the diffuse decline of apparent diffusion coefficient (ADC)

Department of Radiology (C.-Y. Chen, K.-W. Lee, C.-C. Lee, and S.-C. Chin), National Defense Medical Center and Tri-Service General Hospital, 8, Section 3, Ting-Chow Road, Taipei, Taiwan, Department of Electrical Engineering (H.-W. Chung), National Taiwan University, Taipei, Taiwan, Republic of China, and Department of Radiology (R. A. Zimmerman), The Children's Hospital of Philadelphia, 34th Street, Center Civic Boulevard, Philadelphia, PA, U.S.A. Address correspondence and reprint requests to Dr. C.-Y. Chen, Department of Radiology, National Defense Medical Center and Tri-Service General Hospital, 8, Section 3, Ting-Chow Road, Taipei, Taiwan, Republic of China. on the diffusion MR imaging has not been previously reported.

\section{CASE REPORT}

A 46-year-old male sustained sudden onset of aphasia and subsequent dyspnea after intravenous injection of unknown amount of heroin. He was in a comatose status for 3 weeks. When he was transferred to our hospital, physical examination showed an alert mentality and akinetic mutism. Deep tendon reflexes were increased in four limbs and obvious spastic quadriplegia was shown. The size of the pupils was $3 \mathrm{~mm}$ bilaterally. Abnormal laboratory data showed hemoglobin $11.8 \mathrm{gm} / \mathrm{dl}$ (normal male adult $>13 \mathrm{gm} / \mathrm{dl}$ ), and serum phosphorus $5.7 \mathrm{mg} / \mathrm{dl}$ (normal $2.5-5.0 \mathrm{mg} / \mathrm{dl}$ ).

MR scans obtained on the 25th day after the ictus revealed diffuse high signal intensity of cerebral white matter on long TR turbo spin echo T2-weighted images (Fig. 1A) and decreased signal intensity on conventional spin-echo T1-weighted image. Echoplanar diffusionweighted images showed an increase in signal intensity of bilateral frontoparietal white matter and, to a lesser extent, the occipital periventricular white matter in three orthogonal planes of diffusion gradient pulse sequences (Fig. 1B). The normal anisotropy of the internal capsules at posterior limbs was reduced. The basal ganglia were not involved. The brain stem and cerebellum appeared normal in signal intensity on spin echo and echoplanar diffusion images. The derived ADC map from DW MR imaging showed marked decrease of white matter ADC (averaging $\mathrm{ADC}=48.7 \pm 0.8$; normal white matter $\mathrm{ADC}$ value at that period of time was around $70 \pm 1.2$ obtained from the same MR scanner) (Fig. 1C). The 

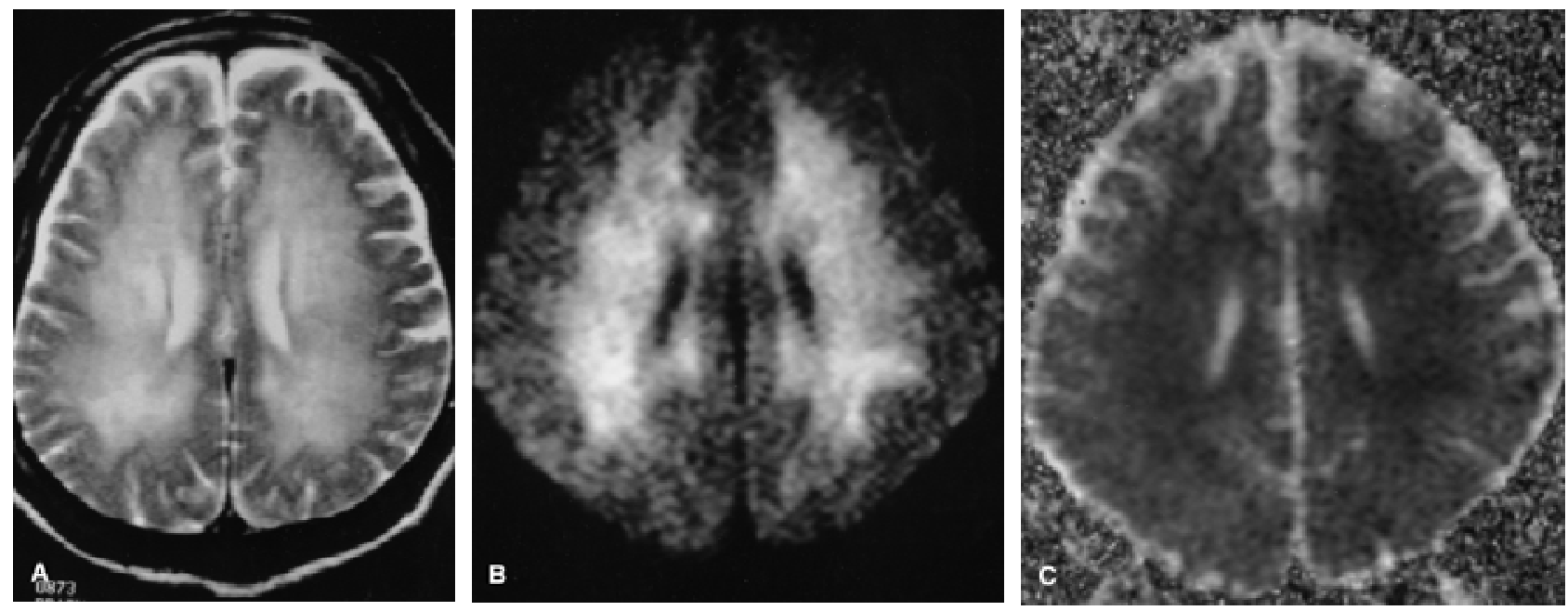

FIG. 1. 46-year-old man with heroin intoxication. A: Axial turbo spin-echo T2-weighted (TR/TE/excitations:4000/99/2) MR image at the level of the upper lateral ventricular body shows diffuse increase in the signal intensity of the hemispheric white matter. B: Axial echoplanar diffusion-weighted (TR/TE/excitations/b value: 4700/118/1/1000) MR image at the same level as Fig. 1A shows generalized increase in signal intensity of the cerebral white matter. C: Corresponding apparent diffusion coefficient (ADC) map to Fig. 1B shows declining ADC of the abnormal white matter.

patient was conservatively treated and then discharged with mild clinical improvement. A follow-up 6 months after his initial ictus revealed complete recovery of neurological function. The patient refused to do the followup MR study.

\section{DISCUSSION}

Focal cerebral lesions revealed by high-signal abnormalities on diffusion-weighted MR images together with a declining ADC have been shown in various diseases, including acute cerebral infarction, cerebral hematoma, Jakob-Creutzfeldt disease, and brain abscess $(7,8)$. It has not been reported that similar DW imaging abnormality may be shown in diffuse white matter disease process, typified by the impaired water diffusion in the myelin sheath. This is probably because most of the leukoencephalopathy and leukodystrophy with demyelination (myelin destruction or myelin pallor) or dysmyelination have microscopic features of diminution or depletion of the myelin (a barrier to water motion) of the white matter axons, resulting in an increase in ADC value and a decrease of diffusion anisotropy (9).

The explanation for the decreased water diffusion of the white matter in our patient can be attributable to the underlying pathologic changes of the myelin in response to heroin-produced neurotoxicity. The neuropathological and electronic microscopic findings of heroin-induced encephalopathy have shown spongiform degeneration of the white matter where vacuoles were formed between the myelin lamellae by splitting of the intraperiod lines (1). Histopathologic evidence of demyelination was lacking in those patients who died soon after the onset of the disease. The accumulation of restricted fluid in between the layers of lamellae of myelin might cause increased isotropy of water diffusion of the white matter and account for the increased signal intensity on the DW MR images, as opposed to the vasogenic white matter edema or hydrocephalus in which fluid accumulation in the white matter interstitium outside the myelin sheaths would increase the water diffusion with resultant decreased anisotropic signal intensity on DW images. On the other hand, because the myelin and blood brain barrier are intact in patients with less severe heroin-induced leukoencephalopathy (1), reversible change of the DW MR signal may be expected. In our patient, the normal or decreased diffusion anisotropy in the brain stem and internal capsules on MR obtained 3 weeks after onset of illness was accompanied with the clinical improvement of symptoms. We suspected that there might have been brain stem and internal capsule involvement in our patient at the acute stage of heroin intoxication.

Although T2-weighted spin-echo MR imaging is sensitive to diffuse white matter disease, it is not specific for diseases with different pathologies such as demyelination, vasogenic white matter edema, and vacuolating myelinopathy (10). In our case, the information provided by DW MR imaging is valuable in that it may suggest the underlying pathological process of the white matter.

\section{REFERENCES}

1. Wolters EC, Van Wijngaarden GK, Stam FC, et al. Leucoencephalopathy after inhaling "heroin" pyrolysate. Lancet 1982;2:1233-6.

2. Weber W, Henkes H, Moller P, et al. Toxic spongiform leucoencephalopathy after inhaling heroin vapour. Eur Radiol 1998;8: 749-55.

3. Boyadjiev S; Pratt VM, Taylor S, et al. Adult-onset neurodegenerative disorder due to proteolipid protein gene mutation in the 
mother of a man with Pelizaeus-Merzbacher disease. Neurology 1996;47:1333-5.

4. Drayer BP. Imaging of the aging brain, part II. Pathologic conditions. Radiology 1988;166:797-806.

5. Rutherford MA, Cowan FM, Manzur AY, et al. MR imaging of anisotropically restricted diffusion in the brain of neonates and infants. J Comput Assist Tomogr 1991;15:188-98.

6. Ono J, Harada K, Mano T, et al. Differentiation of dys- and demyelination using diffusional anisotropy. Pediatr Neurol 1997;16: 63-6.

7. Hajnal JV, Doran M, Hall AS, et al. MR imaging of anisotropically restructed diffusion of water in the nervous system: technical, ana- tomic, and pathologic considerations. J Comput Assist Tomogr 1991;15:1-18.

8. Kim YJ, Chang KH, Song IC, et al. Brain abscess and necrotic or cystic brain tumor: discrimination with signal intensity on diffusion-weighted MR imaging. AJR Am J Roentgenol 1998;171: 1487-90.

9. Hanyu H, Shindo H, Kakizaki D, et al. Diffusion MRI study of cerebral white matter lesions in patients with Binswanger's disease. Rinsho Shinkeigaku 1996;36:442-50.

10. Ay H, Buonanno FS, Schaefer PW, et al. Posterior leukoencephalopathy without severe hypertension: utility of diffusion-weighted MRI. Neurology 1998;51:1369-76. 